\title{
Image-guided thoracic surgery in the hybrid operation room
}

\author{
Hideki Ujiie, Andrew Effat, Kazuhiro Yasufuku
}

Division of Thoracic Surgery, Toronto General Hospital, University Health Network, University of Toronto, Toronto, ON, Canada

Contributions: (I) Conception and design: H Ujiie, K Yasufuku; (II) Administrative support: K Yasufuku; (III) Provision of study materials or patients: K Yasufuku; (IV) Collection and assembly of data: H Ujiie, K Yasufuku; (V) Data analysis and interpretation: H Ujiie, K Yasufuku; (VI) Manuscript writing: All authors; (VII) Final approval of manuscript: All authors.

Correspondence to: Kazuhiro Yasufuku, MD, PhD. Division of Thoracic Surgery, Toronto General Hospital, University of Toronto, 200 Elizabeth St, 9N-957, Toronto, ON M5G2C4, Canada. Email: kazuhiro.yasufuku@uhn.ca.

\begin{abstract}
There has been an increase in the use of image-guided technology to facilitate minimally invasive therapy. The next generation of minimally invasive therapy is focused on advancement and translation of novel image-guided technologies in therapeutic interventions, including surgery, interventional pulmonology, radiation therapy, and interventional laser therapy. To establish the efficacy of different minimally invasive therapies, we have developed a hybrid operating room, known as the guided therapeutics operating room (GTx OR) at the Toronto General Hospital. The GTx OR is equipped with multi-modality image-guidance systems, which features a dual source-dual energy computed tomography (CT) scanner, a robotic conebeam CT (CBCT)/fluoroscopy, high-performance endobronchial ultrasound system, endoscopic surgery system, near-infrared (NIR) fluorescence imaging system, and navigation tracking systems. The novel multimodality image-guidance systems allow physicians to quickly, and accurately image patients while they are on the operating table. This yield improved outcomes since physicians are able to use image guidance during their procedures, and carry out innovative multi-modality therapeutics. Multiple preclinical translational studies pertaining to innovative minimally invasive technology is being developed in our guided therapeutics laboratory (GTx Lab). The GTx Lab is equipped with similar technology, and multimodality image-guidance systems as the GTx OR, and acts as an appropriate platform for translation of research into human clinical trials. Through the GTx Lab, we are able to perform basic research, such as the development of image-guided technologies, preclinical model testing, as well as preclinical imaging, and then translate that research into the GTx OR. This OR allows for the utilization of new technologies in cancer therapy, including molecular imaging, and other innovative imaging modalities, and therefore enables a better quality of life for patients, both during and after the procedure. In this article, we describe capabilities of the GTx systems, and discuss the first-in-human technologies used, and evaluated in GTx OR.
\end{abstract}

Keywords: Lung cancer; hybrid operating room; image-guided therapy; minimally invasive therapy; videoassisted thoracoscopic surgery (VATS)

Received: 06 July 2017; Accepted: 15 September 2017; Published: 28 October 2017.

doi: 10.21037/jovs.2017.09.07

View this article at: http://dx.doi.org/10.21037/jovs.2017.09.07

\section{Development of the guided therapeutics operating room (GTx OR)}

The Guided Therapeutics (GTx) Surgery Program (http://gtxsurgeryprogram.technainstitute.com/) is part of the Guided Therapeutics Program in the Techna Institute (http://technainstitute.com/) of the University
Health Network (UHN). The GTx Program focuses on improvement and translation of advanced imaging and guidance technologies for therapeutic interventions. This includes surgery, interventional pulmonology, radiation therapy, and interventional laser therapy. Although there have been improvements in the detection of small peripheral pulmonary nodules using low-dose computed tomography 


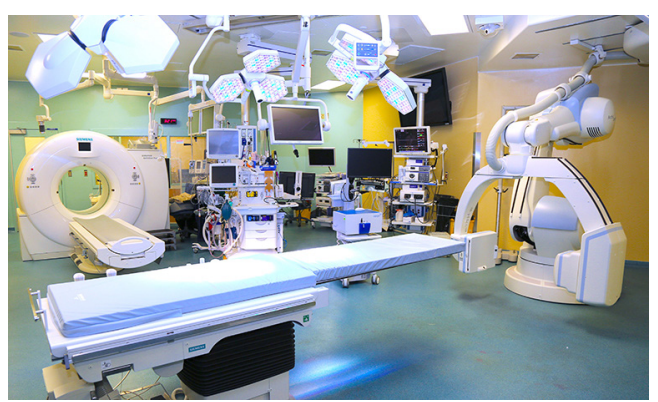

Figure 1 Guided therapeutics (GTx) operation room in Toronto General Hospital. (I) The largest hybrid operating room in Canada; (II) first facility in the world that has a dual sourcedual energy computed tomography (CT) scanner (SOMATOM Definition flash, Siemens) and a state-of-the-art robotic conebeam CT (CBCT)/ fluoroscopy (Artis Zeego, Siemens), within the same OR; (III) equipped with image-based surgical navigation technologies (X-ray and optical navigation; visualization). OR, operating room.

(CT) screening, surgeons are still faced with the difficulties of localization of a small, non-visible, non-palpable pulmonary nodule during video-assisted thoracoscopic surgery (VATS), and robotic-assisted thoracoscopic surgery (RATS). The primary focus of the GTx surgery program is the development of real-time three-dimensional (3D) imaging, and real-time image guided therapy combined with a navigation system, allowing unique therapeutics delivery.

In order to translate this into practice, we have established a hybrid operating room known as the GTx OR at the Toronto General Hospital $(1,2)$. The GTx OR is a state-ofthe-art facility for testing and building devices for surgical guidance. The environment which is populated with novel equipment, as well as surgical, and engineering staff, is set up as an ideal platform to evaluate novel technologies, help establish the appropriate workflow, and human factors considerations needed to allow for integration with existing technology and protocols in clinical practice. The size of the GTx OR is around 1,200 square feet; it is around three times larger than a standard operating room in most modern hospitals, and incorporates the multimodality image-guidance system. The multi-modality image-guidance system features a dual source-dual energy CT scanner (SOMATOM Definition flash, Siemens), a robotic cone-beam CT (CBCT)/fluoroscopy (Artis Zeego, Siemens, Munich, Germany), high-performance endobronchial ultrasound system and surgical endoscopy
(EVIS EXERA III, Olympus, Tokyo, Japan), near-infrared (NIR) fluorescence imaging system (PINPOINT endoscopic fluorescence imaging system, Novadaq, Mississauga, Canada), and navigation tracking systems (SPiN Thoracic Navigation System, Veran, St. Louis, USA) to capture tumors. Our GTx OR is the first site in the world to have these different technologies in a single operating room (Figure 1). The topof-the-line medical imaging systems will enable physicians to quickly, and accurately image patients while they are on the operating table, allowing for real-time feedback to the surgeons, and yielding improved outcomes for the patients.

\section{The capabilities of the guided therapeutics laboratory (GTx Lab)}

The goal of the GTx surgery program is the translation of preclinical research to human clinical trial. The first step of that process is the development of new technology through preclinical studies in the GTx Lab. This feat is possible because of the team's unique multi-corrective research collaboration among scientists, engineers, and physicians with a shared perception toward innovative cancer interventions. The GTx Lab also boasts several technologies that allow for advanced research to take place. This includes: a pair of pre-clinical animal operating rooms (OR) equipped with an endoscopic surgery system (Olympus), an electromagnetic (EM) navigation system developed in-house, a pre-clinical NIR fluorescence imaging system (SPY fluorescence imaging system, and PINPOINT endoscopic fluorescence imaging system, Novadaq), a micro-CT (Locus Ultra, GE Healthcare Life Sciences, Issaquah, USA) for small animal pre-clinical evaluation studies, CBCT (ARCADIS, Siemens) for large animal pre-clinical evaluation studies, and a pre-clinical 1.5T MRI (MAGNETOM Aera, Siemens) (3-8).

The application of these imaging, and navigation techniques has improved the accuracy of resections, improved targeting of cancers, and minimized the unintended impact on surrounding tissue. Initial trials, to validate the benefits of the above-mentioned procedures, were conducted in the GTx Lab on animal models, and cadavers. The surgical procedures, and equipment were then brought into clinical settings of the GTx OR. The GTx team tested the value of its new technology in a clinical trial, and demonstrated significant improvements in accuracy through their equipment. These procedures will be translated from the GTx Lab to first in-human clinical trials in the GTx OR. 
Table 1 Guided therapeutics (GTx) studies in Toronto General Hospital

\begin{tabular}{|c|c|}
\hline GTx studies in Toronto General Hospital & Clinical Trial No. \\
\hline Technique for localization of pulmonary nodules using the spy thoracoscope system (1) & NCT02090660 \\
\hline Cone-beam CT Guided Microcoil Localization of Pulmonary Nodules During Video Assisted Thoracic Surgery (VATS) & NCT02496624 \\
\hline Feasibility of a minimally invasive thoracoscopic ultrasound for localization of pulmonary nodules (9) & NCT02665078 \\
\hline Study Using Combined Virtual 4-D Electromagnetic (EM) Tip-Tracked Devices \& EBUS in Diagnosis of Lung Nodules & NCT01947530 \\
\hline
\end{tabular}

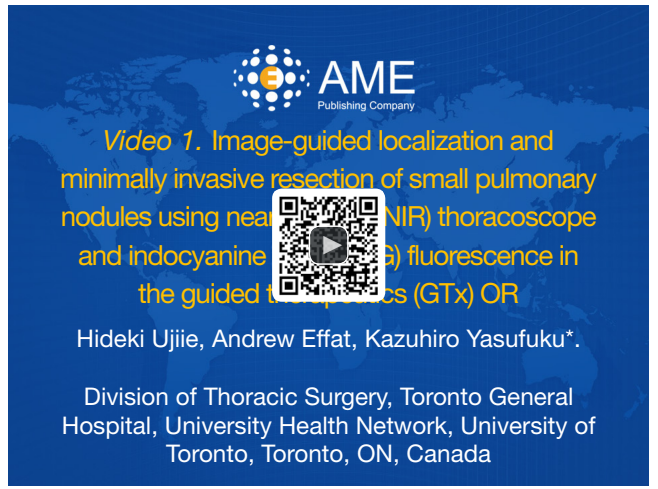

Figure 2 Image-guided localization and minimally invasive resection of small pulmonary nodules using near-infrared (NIR) thoracoscope and indocyanine green (ICG) fluorescence in the guided therapeutics (GTx) OR (10). (I) GTx OR in Toronto General Hospital; (II) preoperative CT-guided microcoil placement and ICG injection; (III) intraoperative NIR localization of small lung nodules.

Available online: http://www.asvide.com/articles/1761

\section{Prospective clinical trials in the GTx OR}

With approval from the Institutional Review Board, we are performing 5 prospective clinical trials in GTx OR $(1,9)$ (Table 1). We briefly discuss each study in this article.

(I) Technique for localization of pulmonary nodules using the spy thoracoscope system (NCT02090660) (https://clinicaltrials. gov/ct2/show/NCT02090660?term=02090660\&rank=1) (1)

We described a new multimodality approach for imageguided localization and minimally invasive resection of small pulmonary nodules using NIR thoracoscope and indocyanine green (ICG) fluorescence in the GTx OR (Figure 2) (1). The standard procedure at our institution for intraoperative localization of small sized lung nodules is CT-guided metallic microcoil placement performed prior to VATS (2,11-13). Preoperative CT-guided placement of a metallic object with intraoperative fluoroscopic assistance has become the common method of localizing small pulmonary nodules, and has been highly successful in localizing nodules with success rates greater than $97 \%$ (2,11-13). However, the metal tag placement process requires a preoperative invasive procedure that can result in patient discomfort, and complications such as metal tag dislodgement, pneumothorax, and hemoptysis (2).

To mitigate these issues, we examined other, less invasive, tumor localization methods $(1,3,4)$. NIR imaging using ICG fluorescence has several unique advantages compared with other localization techniques. The excitation of ICG by a class 3 laser light, and the deep tissue penetration properties of NIR emission light are sufficient for the visualization of the small amount of diluted ICG within the lung parenchyma. This technique does not require radiation exposure. The energy required for exciting the NIR contrast agents is low $\left(10^{-1} \mathrm{eV}\right)$; it has less energy than the lights in an operating room, thus it is safe for humans without shielding (1). In addition, unlike color dye detection by a white light endoscope, the specific wavelength of ICG fluorescence is always detectable regardless of any changes in color or texture of the visceral pleura, which may occur due to anthracosis or other underlying pulmonary diseases. Additionally, ICG fluorescence imaging is easy to understand, and provides a real-time intuitive image for the observer to interpret. Furthermore, ICG is safe and does not necessarily have to be removed from lung tissue. This characteristic offers an advantage compared to other fiducial markers that must be removed together with the pulmonary nodules during surgery, including liquid markers such as radiotracer, iodine, and barium.

In a preclinical study at GTx Lab, we demonstrated a novel NIR thoracoscopic lung nodule localization technique using an injection of fluorescent ICG in vitro, and in vivo swine study (3). We then translated this novel technique 
into a clinical setting through a study in the GTx OR. At first, under dual source-dual energy CT guidance, the microcoil was deployed entirely proximal to the nodule. After deployment of the coil, diluted ICG was injected. After confirmation of satisfactory microcoil position on the CT, the patient was comfortably transferred to the CBCT surgical table and underwent induction with general anesthesia. VATS was initiated, and the NIR thoracoscope was used to localize the nodule with ICG fluorescence. X-ray fluoroscopy was then used to confirm the location of the microcoil to carry out the VATS wedge resection. In this study, we demonstrated that ICG fluorescence localization for resection of small pulmonary nodules with NIR fluorescence thoracoscope is a safe, and feasible minimally invasive approach for localization, and resection of small and ground glass nodules. The use of the GTx OR allowed us the ability to perform a series of surgical procedures without the need to transfer the patient to any other room, ultimately supporting improved workflow throughout the procedure.

(II) Cone-beam CT Guided Microcoil Localization of Pulmonary Nodules During Video Assisted Thoracic Surgery (VATS) (NCT02496624) (https://clinicaltrials.gov/ct2/ show/NCT02496624?term $=02496624 \&$ rank=1)

One of the key goals of the GTx surgery program is to fund and support research for early tumor detection, when tumors are at their smallest and most treatable stages (14). Innovations within the GTx Lab led to the development of a transportable, intra-operative CBCT that has been established in prospective clinical trials for head and neck surgery in our institution (15). CBCT scanners are adept at capturing small tumors, and other surgical targets with unmatched precision. They are also capable of providing GPS-like guidance to the physicians on the safest and most optimal route to the target area. The robotic CBCT is also capable of generating a $3 \mathrm{D}$ image of the surgical site to provide real time, on-the-table imaging, allowing us to update the images as the operation progresses. Combined with some potent computer graphics programming, images of the target lung tumor, surrounding lung parenchyma, and arteries are all projected in multiple formats on two large screens suspended above the operating table. With the GTx's real-time guidance capacity, surgeons have the capability of operating immediately, with increased confidence of finding and removing the target cancer.

The standard procedure at our institution for intraoperative localization of small sized lung nodules is CT-guided metallic microcoil placement performed prior to VATS (2,11-13). Classically, these techniques require localization to be performed in the radiology suite followed by surgery in the OR. On the other hand, the GTx OR does not require transportation of the patient to another operation room for therapy after the imaging. It enables to reduce excessive respiratory motion, excluding potential interruption from anesthetic induction, airway apparatus, and repositioning of patient. The GTx OR currently houses 2 advanced imaging technologies. The first is the dual source-dual energy CT scanner, which has familiar CT image capabilities. The second is the robotic CBCT, which provides excellent 3D imaging for adaptable integration within the operating setting. The integration of these elements into a single OR enables crucial evaluation of the restriction of CT visualization technology for surgical guidance. The centralization of the procedure entirely inside a single OR also removes the concern about complications after the marking interventions (2). These capabilities may have implications in terms of time, and cost savings, as well as a reduction in the rate of microcoil dislodgements.

(III) Study Using Combined Virtual 4-D Electromagnetic (EM) Tip-Tracked Devices \& EBUS in Diagnosis of Lung Nodules (NCT01947530) (https://clinicaltrials.gov/ct2/ show/NCT01947530)

Conventional bronchoscopy techniques have been limited in the evaluation of the peripheral pulmonary nodules, with diagnostic yields. Additional technology, such as radial probe endobronchial ultrasound, and navigational bronchoscopy, have allowed for improved diagnostic yield in the bronchoscopy evaluation of small, peripheral pulmonary nodules. These technologies are an encouraging sign that peripheral pulmonary lesions, inside or close to the bronchial airway, will be accessible through conventional bronchoscopy.

These encouraging signs have led us to develop a preclinical EM navigational bronchoscopy system in the GTx Lab $(3,4)$. The first step of EM navigation bronchoscopy is imaging to create a map of the chest. A CT chest scan is taken using a mobile CBCT, and a $3 \mathrm{D}$ CT image of the thorax is produced. This imaging system is capable of submillimeter spatial resolution, and softtissue visibility at low radiation doses (15). EM navigation devices are then used to track the coordinates of the tip of the flexible endoscope. The cylindrical electromagnetic sensor (5-mm long, 0.8-mm diameter) (Aurora, Northern Digital, Waterloo, Canada) provides 6 degrees of freedom $(x, y, z$, pitch, yaw, roll). The sensor was integrated in the 
tip of the bronchoscope, which is a modification on the original model BF-MP160F (Olympus) (3). EM navigation bronchoscopic images are then registered to the 3D-CBCT images using in-house developed software for image-guided surgical procedures (16). The software provides image data processing and visualization, tool navigation tracking, and rigid image registration algorithms necessary for the superimposition of the bronchoscopic to 3D-CBCT images. The software presents orthogonal (axial, coronal, sagittal) views from the perspective of the tracking device, and real and virtual endoscopic views, where the virtual view is based on the camera position defined by the tracking device. Each of these viewing windows can allow for the overlaying of multiple layers of image data with varying degrees of transparency. The bronchoscopic path is then manually superimposed on the CBCT image by selecting points in the image starting from the tumor location, and proceeding through the bronchus to the trachea. The operator is able to manipulate the bronchoscope by simultaneously viewing both, the real time white light bronchoscopic images, and the navigation images reconstructed using CBCT data. The mapped path is then displayed on the real, and virtual images to provide guidance to the clinician $(3,4,16)$.

We have transferred this technology to a human prospective clinical trial. There are already clinically available products for bronchoscopic EM navigation technology, such as the SPiN Thoracic Navigation System (Veran). We are using this EM navigation bronchoscope system with CBCT fluoroscopy to perform biopsy for peripheral lung tumor. The purpose of this study is to determine the safety, and biopsy yield of an EM navigation bronchoscopic system compared to standard bronchoscopy.

EM navigation bronchoscopy can also provide a lessinvasive approach to preoperative localization of nodule marking, or insertion of a fiducial marker for targeting an excisional biopsy, and radiation therapy. This technology allows the surgeon to minimize unnecessary resection of healthy lung tissue in patients with poor pulmonary function, and other comorbidities, without compromising the ability to make a proper diagnosis, and collecting sufficient tissue for driver mutations, and immune histochemical chemistry. Outside of playing a role in diagnostic procedures, this technology could also provide support for future endobronchial therapeutic technologies such as endobronchial laser therapy (5), thermal ablation therapy, and the injection of chemotherapeutic agents. Therefore, we believe that EM navigation technology is a key component of our hybrid OR because of its potential use for minimally invasive therapies in near future.

These trials will examine the role of intra-operative imaging, and guidance on surgical precision and accuracy. The trials and collaborations will also aid further technical developments of tracking, navigation and image analysis tools.

\section{Conclusions}

The GTx OR is one of the world's first research-based operating rooms. It is a unique platform that will allow for translation from the laboratory into the clinical setting. It will allow for more rapid advancements in interventional therapeutics, including minimally-interventional surgical techniques, robotic surgery procedures, and improved workflow within different surgical procedures. This OR promises a better quality of life for patients, both during and after treatment, and supports the application of new technologies in cancer surgery.

\section{Acknowledgements}

The authors would like to thank Ms. Judy McConnell, Ms. Alexandria Grindlay and Ms. Kimberley Hudson, (Toronto General Hospital) for coordinating prospective clinical research and laboratory management. $\mathrm{H}$ Ujiie received a research scholarship from the Joseph M. West Family Memorial Fund.

\section{Footnote}

Conflicts of Interest: The authors have no conflicts of interest to declare.

\section{References}

1. Ujiie H, Kato T, Hu HP, et al. A novel minimally invasive near-infrared thoracoscopic localization technique of small pulmonary nodules: A phase I feasibility trial. J Thorac Cardiovasc Surg 2017;154:702-11.

2. Kidane B, Yasufuku K. Advances in Image-Guided Thoracic Surgery. Thorac Surg Clin 2016;26:129-38.

3. Anayama T, Qiu J, Chan H, et al. Localization of pulmonary nodules using navigation bronchoscope and a near-infrared fluorescence thoracoscope. Ann Thorac Surg 2015;99:224-30.

4. Wada H, Hirohashi K, Anayama T, et al. Minimally invasive electro-magnetic navigational bronchoscopy- 
integrated near-infrared-guided sentinel lymph node mapping in the porcine lung. PLoS One 2015;10:e0126945.

5. Jin CS, Wada H, Anayama T, et al. An Integrated Nanotechnology-Enabled Transbronchial Image-Guided Intervention Strategy for Peripheral Lung Cancer. Cancer Res 2016;76:5870-80.

6. Ujiie H, Kato T, Hu HP, et al. Development of a novel ex vivo porcine laparoscopic Heller myotomy and Nissen fundoplication training model (Toronto lap-Nissen simulator). J Thorac Dis 2017;9:1517-24.

7. Patel P, Wada H, Hu HP, et al. First Evaluation of the New Thin Convex Probe Endobronchial Ultrasound Scope: A Human Ex Vivo Lung Study. Ann Thorac Surg 2017;103:1158-64.

8. Patel P, Kato T, Ujiie H, et al. Multi-Modal Imaging in a Mouse Model of Orthotopic Lung Cancer. PLoS One 2016;11:e0161991.

9. Ujiie H, Kato T, Hu HP, et al. Evaluation of a New Ultrasound Thoracoscope for Localization of Lung Nodules in Ex Vivo Human Lungs. Ann Thorac Surg 2017;103:926-34.

10. Ujiie H, Effat A, Yasufuku K. Image-guided localization and minimally invasive resection of small pulmonary nodules using near-infrared (NIR) thoracoscope and indocyanine green (ICG) fluorescence in the guided

doi: 10.21037/jovs.2017.09.07

Cite this article as: Ujiie H, Effat A, Yasufuku K. Imageguided thoracic surgery in the hybrid operation room. J Vis Surg 2017;3:148. therapeutics (GTx) OR. Asvide 2017;4:448. Available online: http://www.asvide.com/articles/1761

11. Mayo JR, Clifton JC, Powell TI, et al. Lung nodules: CT-guided placement of microcoils to direct videoassisted thoracoscopic surgical resection. Radiology 2009;250:576-85.

12. Kha LC, Hanneman K, Donahoe L, et al. Safety and Efficacy of Modified Preoperative Lung Nodule Microcoil Localization Without Pleural Marking: A Pilot Study. J Thorac Imaging 2016;31:15-22.

13. Donahoe LL, Nguyen ET, Chung TB, et al. CT-guided microcoil VATS resection of lung nodules: a single-centre experience and review of the literature. J Thorac Dis 2016;8:1986-94.

14. Ujiie H, Kadota K, Chaft JE, et al. Solid Predominant Histologic Subtype in Resected Stage I Lung Adenocarcinoma Is an Independent Predictor of Early, Extrathoracic, Multisite Recurrence and of Poor Postrecurrence Survival. J Clin Oncol 2015;33:2877-84.

15. King E, Daly MJ, Chan H, et al. Intraoperative conebeam CT for head and neck surgery: feasibility of clinical implementation using a prototype mobile C-arm. Head Neck 2013;35:959-67.

16. Weersink RA, Qiu J, Hope AJ, et al. Improving superficial target delineation in radiation therapy with endoscopic tracking and registration. Med Phys 2011;38:6458-68. 